\title{
Electronic structure of semiconductor nanoparticles from stochastic evaluation of imaginary-time path integral
}

\author{
Andrei Kryjevski $\odot^{*}$ \\ Department of Physics, North Dakota State University, Fargo, North Dakota 58108, USA \\ Thomas Luu $\odot^{\dagger}$ \\ Institut für Kernphysik \& Institute for Advanced Simulation, Forschungszentrum Jülich, 54245 Jülich, Germany \\ Valentin Karasiev \\ Laboratory for Laser Energetics, University of Rochester, Rochester, New York 14623, USA
}

(Received 29 May 2020; revised 19 August 2020; accepted 3 May 2021; published 4 June 2021)

\begin{abstract}
The fermion sign problem, when severe, prevents the computation of physical quantities of a system of interacting fermions via stochastic evaluation of its path integral. This is due to the oscillatory nature of the integrand $\exp (-S)$, where $S$ is the imaginary-time action. This issue is a major obstacle to first-principles lattice quantum Monte Carlo studies of excited states of electrons in matter. However, in the Kohn-Sham orbital basis, which is the output of a density-functional theory simulation, the path integral for electrons in a semiconductor nanoparticle has only a mild fermion sign problem and is amenable to evaluation by standard stochastic methods. This is evidenced by our simulations of silicon hydrogen-passivated nanocrystals such as $\mathrm{Si}_{35} \mathrm{H}_{36}, \mathrm{Si}_{87} \mathrm{H}_{76}, \mathrm{Si}_{147} \mathrm{H}_{100}$, and $\mathrm{Si}_{293} \mathrm{H}_{172}$, which range in size $1.0-2.4 \mathrm{~nm}$ and contain 176 to 1344 valence electrons. We find that approximating the fermion action by its leading order polarization term results in a positive-definite integrand in the functional integral, and is a very good approximation of the full action. We compute imaginary-time electron propagators and extract the energies of low-lying electron and hole levels. Our quasiparticle gap predictions agree with the results of previous high-precision $G_{0} W_{0}$ calculations. This formalism allows calculations of more complex excited states such as excitons and trions.
\end{abstract}

DOI: 10.1103/PhysRevResearch.3.023173

Applications of semiconductor nanomaterials require quantitative understanding of their electronic structure including excited state properties. In recent years computational studies of atomistic models of these systems using $a b$ initio electronic structure techniques have proven to be an attractive alternative to actual experiments as the ability to explore the vast set of possible configurations is inevitably limited. Currently, density-functional theory (DFT) [1,2] is the most useful first-principles electronic structure method. It combines reasonable accuracy and applicability, and naturally allows inclusion of surfaces, interfaces, dopants, ligands, etc.

However, DFT predicts ground state properties. Therefore, alternative methods are required to study the excited states. Several approaches, such as Moller-Plesset perturbation theory [3,4], configuration interaction [5], coupled clusters [6], dynamical mean-field theory [7], time-dependent DFT

\footnotetext{
*andrei.kryjevski@ndsu.edu

†tluu@fz.juelich.de

‡vkarasev@1le.rochester.edu
}

Published by the American Physical Society under the terms of the Creative Commons Attribution 4.0 International license. Further distribution of this work must maintain attribution to the author $(s)$ and the published article's title, journal citation, and DOI.
[8,9], two-electron reduced density matrix method [10,11], etc., have been developed. Currently, the most efficient comprehensive $a b$ initio technique is based on many-body perturbation theory (MBPT), where DFT is augmented by the methods of perturbative many-body quantum mechanics. For instance, the GW method is used to compute single-particle energies, the Bethe-Salpeter equation (BSE) is solved for exciton states; a technique for three-body states-trions-also exists $[9,12-15]$. The resulting energies and wave functions are subsequently used in calculations of various excited-state properties (e.g., [16-18]).

On the other hand, calculating physical quantities from the correlation functions using stochastic evaluation of the path integral defined on a discretized space-time domain is a standard nonperturbative method for interacting fermions (see, e.g., [19-22] and references within), which is the lattice field theory approach, also called (determinantal) quantum Monte Carlo (QMC) [23]. While very successful in, e.g., lattice quantum chromodynamics (QCD) at zero baryon density, this approach typically fails for dense Fermi systems [23,24], including electrons in a nanoparticle. This is due to the fermion sign problem (FSP) $[25,26]$. Note that QMC techniques, such as fixed node, diffusion, and auxiliary field, for electronic systems have been developed (see, e.g., [27-30]). Here the FSP is mild enough to allow simulations, but these methods are mostly suited for studying the ground state. Work on a QMC 
technique for excited states has started, but the studies so far are based on the tight-binding approximation and applied to model systems (see, e.g., $[31,32]$ ) and to graphene and carbon nanotubes, which are sign problem free [33-40].

So, a first-principles nonperturbative approach for excited states in a generic semiconductor nanostructure is currently lacking. Therefore, here we present a DFT-based comprehensive QMC technique for a semiconductor nanoparticle, where excited states, such as electrons and holes, excitons and trions, can be obtained from the output of the same MC simulation. The system-specific Kohn-Sham (KS) orbitals are used as a basis in the electron action in the path integral representation of the statistical sum. Our results suggest that in this approach there is only a mild FSP and evaluation by the standard stochastic importance sampling methods employed in, e.g., lattice QCD (see [21] and references within) is possible. Specifically, we present results of simulations of several semiconductor nanocrystals (NCs), such as $\mathrm{Si}_{35} \mathrm{H}_{36}, \mathrm{Si}_{87} \mathrm{H}_{76}, \mathrm{Si}_{147} \mathrm{H}_{100}$, and $\mathrm{Si}_{293} \mathrm{H}_{172}$, including low-lying single-particle energies.

In the Born-Oppenheimer approximation the nonrelativistic Hamiltonian for valence electrons is

$$
\begin{aligned}
\mathrm{H}= & \int d \mathbf{x} \psi_{\alpha}^{\dagger}\left(-\frac{\hbar^{2} \nabla^{2}}{2 m}+e V_{e N}+A_{0}\right) \psi_{\alpha} \\
& +\int \frac{d \mathbf{x}}{8 \pi e^{2}}\left(\vec{\nabla} A_{0}\right)^{2} .
\end{aligned}
$$

Here $\psi_{\alpha}(\mathbf{x})$ is the electron field operator, $\alpha$ is the spin index, $e$ is the electron charge, $V_{e N}\left(\mathbf{x}, \mathbf{R}_{I}\right)$ is a pseudopotential, i.e., an effective potential of ions at positions $\mathbf{R}_{I}$ felt by the valence electrons [41,42]; $A_{0}(\mathbf{x})$ is the scalar potential operator which mediates electron electrostatic interactions; here $A_{0}$ has energy units. The $A_{0}$ terms can be integrated out leading to the standard two-body Coulomb interaction operator, but Eq. (1) is more suitable for our purposes.

The KS equation of the orbital-based DFT with a semilocal exchange-correlation functional, such as that by Perdew, Burke, and Ernzerhof (PBE) [43], is

$$
\begin{aligned}
\left(-\frac{\hbar^{2}}{2 m} \nabla^{2}+e V_{e N}+e V_{K S}\right) \phi_{i}(\mathbf{x}) & =\epsilon_{i} \phi_{i}(\mathbf{x}), \\
V_{K S}=V_{H}+V_{x c}, V_{H}(\mathbf{x}) & =\int d \mathbf{x}^{\prime} \frac{e n\left(\mathbf{x}^{\prime}\right)}{\left|\mathbf{x}-\mathbf{x}^{\prime}\right|}, \\
V_{x c}(\mathbf{x}) & =\frac{\delta E_{x c}[n]}{e \delta n(\mathbf{x})},
\end{aligned}
$$

where $\phi_{i}(\mathbf{x}), \epsilon_{i}$ are the KS orbitals and eigenvalues, respectively, and $E_{x c}[n]$ is the exchange-correlation functional, and $n(\mathbf{x})$ is the ground-state density of valence electrons $[2,43,44]$. In general, the state label $i$ may include band number, lattice wave vector, and spin label. But in this work we only consider spin-symmetric aperiodic systems so that $\phi_{i \uparrow}(\mathbf{x})=$ $\phi_{i \downarrow}(\mathbf{x}) \equiv \phi_{i}(\mathbf{x})$. Extension to the case of a periodic and/or spin-polarized system is straightforward.

In order to utilize electronic structure information from the DFT output we introduce $a_{i \alpha}$, which is the annihilation operator of a fermion in the KS state $|i, \alpha\rangle$; then $\psi_{\alpha}(\mathbf{x})=$ $\sum_{i} \phi_{i}(\mathbf{x}) \mathrm{a}_{i \alpha}$ (see, e.g., [45,46]). In terms of $\mathrm{a}_{i \alpha}$, i.e., in the KS orbital basis, the imaginary-time (Euclidean) action corresponding to the Hamiltonian (1) is

$$
\begin{aligned}
S_{E}= & \int_{0}^{\beta} d \tau\left(\sum_{i j \alpha} a_{i \alpha}^{\dagger}(\tau)\left[\left(\partial_{\tau}+\epsilon_{i}-\mu\right) \delta_{i j}-V_{i j}^{x c}+\mathrm{i} A_{i j}\right] a_{j \alpha}(\tau)\right. \\
& \left.-\mathrm{i} \int d \mathbf{x} A_{0} n(\mathbf{x})+\int \frac{d \mathbf{x}}{8 \pi e^{2}}\left(\vec{\nabla} A_{0}\right)^{2}\right),
\end{aligned}
$$

where $\beta=1 / T_{e}, T_{e}$ is the electronic temperature in energy units used in the simulation, $T_{e} \ll E_{g}$, where $E_{g}$ is the gap, and

$$
\begin{aligned}
V_{i j}^{x c} & =e \int d \mathbf{x} \phi_{i}^{*}(\mathbf{x}) V^{x c}(\mathbf{x}) \phi_{j}(\mathbf{x}), \\
A_{i j}(\tau) & =\int d \mathbf{x} \phi_{i}^{*}(\mathbf{x}) A_{0}(\tau, \mathbf{x}) \phi_{j}(\mathbf{x}) .
\end{aligned}
$$

The spatial integration is over the simulation box volume; KS indices $i$ and $j$ vary over the range of KS orbitals included in the simulation-the "active window". Fermion fields $a_{i \alpha}(\tau)$ are antiperiodic $a_{i}(\tau)=-a_{i}(\tau+\beta)$, while the $A_{0}$ fields are periodic in time. The grand canonical chemical potential $\mu$ is set at the mid-gap, which ensures charge neutrality. In order to obtain (3) we added and subtracted $e V_{K S}(\mathbf{x})$ from (2) to the electron Lagrangian, and then used $\nabla^{2} V_{H}=-4 \pi e n(\mathbf{x})$ combined with the shift-invariance of the functional integration over $A_{0}$. The observables are computed from the functional integral representation of the statistical sum (see, e.g., $[20,47]$ )

$$
Z\left(\mu, T_{e}\right)=\int \mathrm{D} A_{0} \mathrm{D} a_{i} \mathrm{D} a_{i}^{\dagger} \mathrm{e}^{-S_{E}} .
$$

Also, since KS states are labeled by their energy, it is straightforward to only include few KS states near the Fermi level that are relevant to the description of low-energy excitations, which is more efficient than using spatial grid covering the whole simulation cell. Thus, in this approach low-energy excitations of a nanoparticle are described by the KS quasiparticles subject to the static potential $V_{i j}^{x c}$ and interacting via $A_{i j}(\tau)$ exchanges. Here, the FSP may be due to the oscillatory nature of $\exp (-S)$ in Eq. (6) which would preclude importance sampling of the $A_{0}$ configurations.

Strictly speaking, one should proceed using the basis of $\epsilon_{i} \delta_{i j}-V_{i j}^{x c}$ eigenstates. However, setting $V_{i j}^{x c}=V_{i i}^{x c} \delta_{i j}$ is an approximation often made in the GW method $[9,48]$. Also, for the systems we have simulated and for the range of KS states included, $V_{i j}^{x c}$ is strongly dominated by its diagonal entries. So, here we approximate $V_{i j}^{x c}=V_{i i}^{x c} \delta_{i j}$.

Next we perform Grassmann integration over the fermion variables $a^{\dagger}, a$ (see, e.g., [49]) and expand the resulting action $S\left(A_{0}\right)$ in powers of $A_{0}(\tau, \mathbf{x})$ (see, e.g., [20]). The terms linear in $A_{0}$ cancel, which reflects the system's overall charge neutrality. Retaining the leading nonvanishing term in the expansion yields the following action

$$
S_{2}=-\int \frac{d \tau \mathrm{d} \mathbf{x}}{8 \pi e^{2}} A_{0} \nabla^{2} A_{0}-\operatorname{tr} \mathcal{M} \mathcal{A M A}+\mathcal{O}\left(A_{0}{ }^{3}\right),
$$

where

$$
\mathcal{M}_{i j}^{-1}(\tau)=\left(\partial_{\tau}+\tilde{\epsilon}_{i}\right) \delta_{i j}, \tilde{\epsilon}_{i}=\epsilon_{i}-\mu-V_{i i}^{x c}
$$

is the inverse noninteracting propagator and $\mathcal{A}_{i \tau, j \tau^{\prime}}=$ $A_{i j}(\tau) \delta_{\tau \tau^{\prime}}$. The second term in (7) includes the random phase 
approximation (RPA) polarization insertion in the KS orbital basis. The approximate action (7) has been used in this work to simulate the nanoparticle electrons.

But, in order to evaluate the statistical sum (6) numerically we define the action on a discretized space-time grid. The Lagrangian corresponding to Eq. (1) is invariant under timedependent, spatially uniform $U(1)$ gauge transformations

$$
\psi^{\prime}(\tau)=e^{\mathrm{i} \Lambda(\tau)} \psi(\tau), A_{0}^{\prime}=A_{0}-\partial_{\tau} \Lambda(\tau),
$$

where $\Lambda(\tau)$ is a function of time [33]. Note that the gauge field action - the last term in (3) — is invariant under (9) [33] [50]. To generate $A_{0}$-field configurations we have used the frequency representation and a spatial grid, where the polarization term was expressed as a function of $\mathbf{x}, \mathbf{y}$ using $\phi_{j}(\mathbf{x})$. In this case $A_{0}(\omega, \mathbf{x})$ can be used instead of the link variables required on a Euclidean time lattice [51]. However, a Euclidean time-KS grid-a $\tau$-KS lattice-where $\left\{A_{0}(\omega, \mathbf{x})\right\}$ have been converted to the link variables $\exp \left(-\mathrm{i} \delta A_{i j}(\tau)\right)$, where $\delta$ is the $\tau$ grid spacing, are to be used to compute the observables. While more computationally expensive than a $\tau$-KS lattice, the $\omega \mathbf{x}$ basis is used since (1) numerical cancellation of the "tadpole" terms in a simulation requires perfect representation of the time derivative operator $\partial_{\tau}$; (2) the Laplacian in the $A_{0}$-field action cannot be represented accurately with the few $\mathrm{KS}$ orbitals included in a reasonably-sized active window. Then, the action is

$$
S_{2}\left(A_{0}\right)=-\sum_{k \geqslant 0} \sum_{\mathbf{x}, \mathbf{y}} A_{0}^{*}\left(\omega_{k}, \mathbf{x}\right) \mathcal{S}_{k}(\mathbf{x}, \mathbf{y}) A_{0}\left(\omega_{k}, \mathbf{y}\right),
$$

where $\omega_{k}=2 \pi k T_{e}$ is a bosonic Matsubara frequency, and

$$
\begin{aligned}
\mathcal{S}_{k}(\mathbf{x}, \mathbf{y})= & \frac{a_{x} a_{y} a_{z} T_{e}}{8 \pi e^{2}} \nabla_{\mathbf{x}, \mathbf{y}}^{2}+2\left(a_{x} a_{y} a_{z}\right)^{2} \sum_{i j} \phi_{i}^{*}(\mathbf{x}) \phi_{j}(\mathbf{x}) \\
& \times \phi_{j}^{*}(\mathbf{y}) \phi_{i}(\mathbf{y}) \frac{n_{j}-n_{i}}{2 \pi \mathrm{i} k-\beta \tilde{\epsilon}_{j}+\beta \tilde{\epsilon}_{i}}, \\
\nabla_{\mathbf{x}, \mathbf{y}}^{2}= & \sum_{l=1}^{3}\left(\nabla_{l}^{2}\right)_{\mathbf{x}, \mathbf{y}}, \\
\nabla_{l}^{2} f(\mathbf{x})= & \frac{1}{a_{l}^{2}}\left(f\left(\mathbf{x}+a_{l} \hat{l}\right)-2 f(\mathbf{x})+f\left(\mathbf{x}-a_{l} \hat{l}\right)\right)+\mathcal{O}\left(a_{l}^{2}\right),
\end{aligned}
$$

where $a_{l}, l=x, y, z$, are the lattice spacings and $n_{i}=$ $\left(\exp \left[\beta \tilde{\epsilon}_{i}\right]+1\right)^{-1}$. Gauge invariance requires that each included $A(\omega)$ is coupled to at least one pair of fermion modes $a^{\dagger}\left(\omega_{1}\right), a\left(\omega_{2}\right)$ with $\omega=\omega_{2}-\omega_{1}$. Therefore, in the simulations where a finite frequency cutoff was used the expression for $\mathcal{S}_{k}(\mathbf{x}, \mathbf{y})$ was modified accordingly.

Importantly, $\mathcal{S}_{k}(\mathbf{x}, \mathbf{y})$ from Eq. (11) is real and symmetric under $\mathbf{x} \leftrightarrow \mathbf{y}$, which is due to the basic properties of KS eigenfunctions (see, e.g., [52]). Then the action $S_{2}\left(A_{0}\right)$ from (10) is nonnegative, which suggests that in this approach meaningful simulations are possible without resorting to sign-suppression techniques (see, e.g., [53,54]). The size of neglected higherorder terms in the action expansion will be discussed later. The action (10) is quadratic in $A_{0}$. Therefore, importance sampling is done by diagonalizing $\mathcal{S}_{k}(\mathbf{x}, \mathbf{y})$ for each $\omega_{k}$ which results in

$$
S_{2}\left(A_{0}\right)=\sum_{k \geqslant 0} \sum_{i=1}^{N_{x}-1} \frac{\lambda_{i}\left(\omega_{k}\right)}{2}\left(u\left(\omega_{k}\right)_{i}^{2}+v\left(\omega_{k}\right)_{i}^{2}\right),
$$

where $\lambda_{i}\left(\omega_{k}\right)>0, v(0)_{i} \equiv 0, N_{x}$ is the number of spatial grid points. One generates random values for the $u\left(\omega_{k}\right)_{i}, v\left(\omega_{k}\right)_{i}$ variables that are distributed normally according to the corresponding eigenvalue $\lambda_{i}\left(\omega_{k}\right)$, and changes the basis to get the $A_{0}(\tau, \mathbf{x})$ configurations. For all the NCs simulated here $\mathcal{N} \simeq 10^{3}$ configurations have been found to be sufficient to obtain statistically significant results.

The observables considered here are the electron and hole quasiparticle energies, which have been extracted from two-point propagators, which are the averages of the matrix elements of the propagator matrix corresponding to the state $i$ and time slices $\tau_{1}$ and $\tau_{2}$, in the long-time limit. For a particle state $i>H O$, where $H O$ labels the highest occupied orbital, it is $E_{g} \beta \gg E_{g} \tau \gg 1$, then

$$
\begin{aligned}
\mathrm{M}_{i}\left(A_{0}\right)\left(\tau_{2}, \tau_{1}\right) & =\left.\left(\delta\left[\mathrm{D}+\tilde{\epsilon}_{i}\right]\right)^{-1}\right|_{i \tau_{2}, i \tau_{1}}, \\
\mathrm{D}_{i j} f(\tau) & =\frac{1}{\delta}\left(f(\tau+\delta)-e^{-\mathrm{i} \delta A_{i j}(\tau)} f(\tau)\right), \\
\mathrm{M}_{i}(\tau, 0) & =\left\langle\mathrm{M}_{i}\left(A_{0}\right)\right\rangle=\sum_{k=1}^{\mathcal{N}} \frac{\mathrm{M}_{i}\left(A_{0}^{k}\right)}{\mathcal{N}} \rightarrow C e^{-E_{i} \tau},
\end{aligned}
$$

where $\delta=\beta / N_{t}, N_{t}$ is the number of time grid points, $A_{i j}(\tau)$ is defined in (5), $\tilde{\epsilon}_{i}$ is defined in (8). The last line in (13) shows the behavior of the correlator at large times on the fully interacting excitation energy $E_{i}$ ( $C$ is an irrelevant coefficient). We fit for this energy and estimate the statistical error of $E_{i}$ via the bootstrapping technique [55]. A full error analysis, which would include an assessment of systematic errors due to, e.g., fit-window sizes and fitting functions, finite lattice spacings, etc., is left to future work, though we do not expect the uncertainties quoted here to change significantly. We have used time grids with $\delta=0.025 \mathrm{eV}^{-1}$ for all systems after checking that KS energies can be accurately extracted from the propagators in the noninteracting cases. Frequency cutoffs

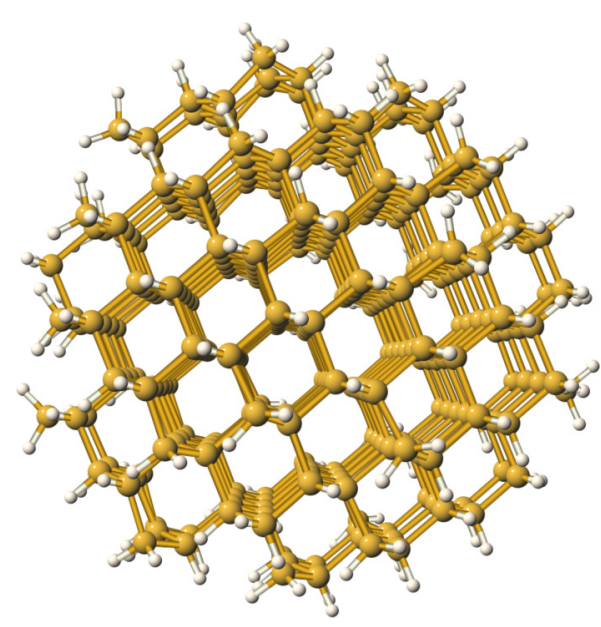

FIG. 1. Atomistic model of $\mathrm{Si}_{293} \mathrm{H}_{172}$. The smaller (white) surface atoms are the hydrogens. 
TABLE I. Both $s$ and $\tilde{s}_{2}$ [defined in Eq. (14)] are dimensionless; $s=|s| e^{i \varphi}$. Numbers in parentheses represent statistical errors. $N_{e}$ is the valence electron number.

\begin{tabular}{lcccc}
\hline \hline & $\mathrm{Si}_{35} \mathrm{H}_{36}$ & $\mathrm{Si}_{87} \mathrm{H}_{76}$ & $\mathrm{Si}_{147} \mathrm{H}_{100}$ & $\mathrm{Si}_{293} \mathrm{H}_{172}$ \\
\hline$T_{e}, \mathrm{eV}$ & 0.5 & 0.5 & 0.4 & 0.3 \\
$N_{e}$ & 176 & 424 & 688 & 1344 \\
$\left\langle\tilde{S}_{2}\right\rangle$ & $74.1(5)$ & $76.2(3)$ & $315.4(3.4)$ & $167.2(3)$ \\
$\operatorname{Re}\langle s\rangle$ & $64.0(3)$ & $70.0(2)$ & $287.9(2.1)$ & $162.2(2)$ \\
$\operatorname{Im}\langle s\rangle$ & $0.1(2)$ & $-0.1(1)$ & $0.2(2)$ & $-0.1(2)$ \\
$\left\langle\tilde{S}_{2} /|s|\right\rangle$ & $1.155(1)$ & $1.0865(7)$ & $1.073(3)$ & $1.0294(3)$ \\
$\langle\tan (\varphi)\rangle$ & $0.002(3)$ & $(-0.6 \pm 2.0) \times 10^{-3}$ & $0.0006(8)$ & $-0.0005(12)$ \\
\hline \hline
\end{tabular}

were chosen so that $\omega_{\max } \delta \simeq 1$. The procedure to extract hole excitation energies is analogous.

In order to generate $\epsilon_{i}, \phi_{i}(\mathbf{x})$ DFT simulations of the atomistic models of the $\mathrm{NCs}$ (such as $\mathrm{Si}_{293} \mathrm{H}_{172}$ shown in Fig. 1) have been done using Quantum Espresso DFT program [56] with the PBE exchange-correlation functional, including geometry relaxation. Norm-conserving pseudopotentials [57] have been used ensuring that the KS orbitals are orthonormal. Kinetic energy cutoff, which determines lattice spacings $a_{i}, i=x, y, z$, has been set to $340.1 \mathrm{eV}$ which is the same as in [48], resulting in $a_{i} \simeq 0.05 \mathrm{~nm}$. The NCs ranging in size from 1.0 to $2.4 \mathrm{~nm}$ were placed in the periodic cubic simulation boxes with about $1 \mathrm{~nm}$ of vacuum between the surfaces in order to prevent spurious interactions between periodic images. The number of KS orbitals included in the simulations have been chosen so that $\epsilon_{i_{\max }}-\epsilon_{\mathrm{HO}} \simeq \epsilon_{\mathrm{HO}+1}-\epsilon_{i_{\min }} \geqslant 1.5 E_{g}^{\mathrm{PBE}}$, where $i_{\max }, i_{\min }$ are the highest and the lowest included $\mathrm{KS}$ orbital labels and $E_{g}^{\mathrm{PBE}}=\epsilon_{\mathrm{HO}+1}-\epsilon_{\mathrm{HO}}$ is the DFT gap. The number of states above/below Fermi level included in the simulation varied from 36 to 96 as the system's size increased.

For the nanoparticles considered in this work $V_{i i}^{x c}$ shifts are sizable and tend to shrink the noninteracting gap $E_{g}^{\mathrm{PBE}}$. This would require lowering $T_{e}$ in order to maintain $E_{g}^{\mathrm{PBE}} \gg T_{e}$ required for the tadpole term cancellation, which would significantly increase the computational expense. So, we have treated $V_{i i}^{x c}$ as self-energy corrections, i.e., we have simulated with $\tilde{\epsilon}_{i}=\epsilon_{i}-\mu$ and subtracted $V_{i i}^{x c}$ from the resulting singleparticle energies.

The main calculation results are shown in Tables I and II. In order to check the size of the terms neglected in the approximate action (10) we have used the A-configurations generated with (10) to compute

$$
\begin{aligned}
s & =\operatorname{tr} \log (1+\mathrm{i} \mathcal{M} \mathcal{A})-\mathrm{i} \operatorname{tr}(\mathcal{M A}), \\
\tilde{s}_{2} & =\frac{1}{2} \operatorname{tr}(\mathcal{M} \mathcal{A} \mathcal{M} \mathcal{A}),
\end{aligned}
$$

TABLE II. All entries are in eV. $E_{g}^{\mathrm{PBE}} \equiv \epsilon_{\mathrm{HO}+1}-\epsilon_{\mathrm{HO}}$, is the PBE gap; the interacting gap is $E_{g}^{\mathrm{QP}}=E_{p}^{\min }-E_{h}^{\max } . G_{0} W_{0}$ results are from [48].

\begin{tabular}{lllll}
\hline \hline & $\mathrm{Si}_{35} \mathrm{H}_{36}$ & $\mathrm{Si}_{87} \mathrm{H}_{76}$ & $\mathrm{Si}_{147} \mathrm{H}_{100}$ & $\mathrm{Si}_{293} \mathrm{H}_{172}$ \\
\hline$E_{g}^{\mathrm{PBE}}$ & 3.51 & 2.59 & 2.29 & 1.79 \\
$E_{g}^{\mathrm{QP}}$ & $6.29(9)$ & $4.76(8)$ & $4.22(6)$ & $3.45(3)$ \\
$E_{g}^{G_{0} W_{0}}$ & 6.29 & 4.77 & 4.21 & 3.46 \\
\hline \hline
\end{tabular}

i.e., the full fermion action $s$ and its leading order approximation $\tilde{s}_{2} ; \mathcal{M}$ and $\mathcal{A}$ are defined in and right after Eq. (8). While $\tilde{s}_{2}$ is only the leading nonvanishing term in the expansion of $s$, we have found that in all cases $\left\langle\tilde{s}_{2} /|s|\right\rangle$ is close to one (see Table I). This suggests that the full fermion action can be reasonably approximated by just the leading term. This is in agreement with the well-known result that in the KS basis the RPA approximation for polarization yields accurate description of semiconductor nanoparticles [9]. The average of the action's phase $\varphi$, where $s=|s| e^{i \varphi}$, is close to zero, which suggests that the sign problem in these systems is mild. Shown in Table II are the quasiparticle gap predictions in these NCs, which agree with the results of high-precision $G_{0} W_{0}$ calculations for the same NCs [48]. Low-energy particle and hole levels in $\mathrm{Si}_{293} \mathrm{H}_{172}$ are shown in Fig. 2.

In conclusion, we have performed initial steps toward development of a first-principles high-precision QMC technique for the excited states of a semiconductor nanoparticle, which utilizes KS orbital basis in the imaginary-time functional integral for the electrons. We find that approximating fermion action with the leading order RPA polarization term in the expansion in powers of $A_{0}$ leads to a positive definite integrand in the statistical sum and that it is a very good approximation to the full action; $\left\langle\tilde{s}_{2} /|s|\right\rangle-1$ can be viewed as a source of systematic error. So, our results suggest that in this approach these systems have only a mild fermion sign problem. Applications of the approximate method based on Eq. (7) to various

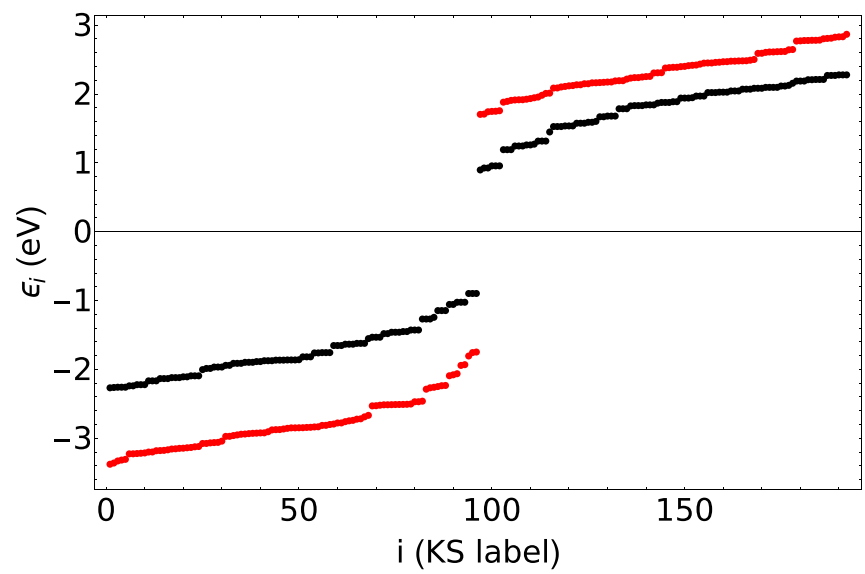

FIG. 2. Interacting quasiparticle energies (red) for $\mathrm{Si}_{293} \mathrm{H}_{172}$. Black points are the KS eigenvalues. Error bars are too small to be seen at this scale. 
semiconductor nanostructures in order to study the range of its applicability by computing $\left\langle\tilde{s}_{2} /|s|\right\rangle$ of Eq. (14) are in progress.

Diagonalization of $\mathcal{S}_{k}(\mathbf{x}, \mathbf{y})$ from Eq. (11) largely determines the overall method's performance. Standard methods scale as $\mathcal{O}\left(N_{x}^{3}\right)$ [58], but implementation of more efficient tools, such as ARPACK [59], TRLan (Thick-Restart Lanczos) [60] programs, is under way.

An obvious method improvement will be to use the full action instead of Eq. (7), which could be done via re-weighting $[61,62]$ or more advanced sampling techniques (e.g., hybrid MC [63]) accompanied by an approximation to suppress FSP in a gauge-invariant way, and followed by a posteriori check of the sign problem severity. Also, development of techniques for other excited states (excitons and trions) is in progress.

The authors acknowledge use of computational resources at the Center for Computationally Assisted Science and Technology (CCAST) at North Dakota State University. T.L. acknowledges financial support from the Deutsche Forschungsgemeinschaft (Sino-German CRC 110). V.K. acknowledges support by the Department of Energy National Nuclear Security Administration under Award No. DE-NA0003856.
[1] P. Hohenberg and W. Kohn, Inhomogeneous electron gas, Phys. Rev. 136, B864 (1964).

[2] W. Kohn and L. J. Sham, Self-consistent equations including exchange and correlation effects, Phys. Rev. 140, A1133 (1965).

[3] Chr. Møller and M. S. Plesset, Note on an Approximation Treatment for Many-Electron Systems, Phys. Rev. 46, 618 (1934).

[4] K. Raghavachari, J. Pople, E. Replogle, and M. Head-Gordon, Fifth order Moeller-Plesset perturbation theory: Comparison of existing correlation methods and implementation of new methods correct to fifth order, J. Phys. Chem. 94, 5579 (1990).

[5] C. Cramer, Essentials of Computational Chemistry: Theories and Models (Wiley, Hoboken, NJ, 2002).

[6] R. J. Bartlett and M. Musiał, Coupled-cluster theory in quantum chemistry, Rev. Mod. Phys. 79, 291 (2007).

[7] G. Kotliar, S. Y. Savrasov, K. Haule, V. S. Oudovenko, O. Parcollet, and C. A. Marianetti, Electronic structure calculations with dynamical mean-field theory, Rev. Mod. Phys. 78, 865 (2006).

[8] E. Runge and E. K. U. Gross, Density-Functional Theory for Time-Dependent Systems, Phys. Rev. Lett. 52, 997 (1984).

[9] G. Onida, L. Reining, and A. Rubio, Electronic excitations: Density-functional versus many-body Green's-function approaches, Rev. Mod. Phys. 74, 601 (2002).

[10] D. Mazziotti, Quantum chemistry without wave functions: Twoelectron reduced density matrices, Acc. Chem. Res. 39, 207 (2006).

[11] S. Hemmatiyan, M. Sajjan, A. W. Schlimgen, and D. A. Mazziotti, Excited-state spectra of strongly correlated molecules from a reduced-density-matrix approach, J. Phys. Chem. Lett. 9, 5373 (2018).

[12] L. Hedin, New method for calculating the one-particle Green's function with application to the electron-gas problem, Phys. Rev. 139, A796 (1965).

[13] M. S. Hybertsen and S. G. Louie, Electron correlation in semiconductors and insulators: Band gaps and quasiparticle energies, Phys. Rev. B 34, 5390 (1986).

[14] M. Rohlfing and S. G. Louie, Electron-hole excitations and optical spectra from first principles, Phys. Rev. B 62, 4927 (2000).

[15] T. Deilmann, M. Drüppel, and M. Rohlfing, Three-Particle Correlation from a Many-Body Perspective: Trions in a Carbon Nanotube, Phys. Rev. Lett. 116, 196804 (2016).
[16] M. Rohlfing and S. G. Louie, Excitonic Effects and the Optical Absorption Spectrum of Hydrogenated Si Clusters, Phys. Rev. Lett. 80, 3320 (1998).

[17] C. D. Spataru, S. Ismail-Beigi, L. X. Benedict, and S. G. Louie, Excitonic Fffects and Optical Spectra of Single-Walled Carbon Nanotubes, Phys. Rev. Lett. 92, 077402 (2004).

[18] A. Kryjevski, D. Mihaylov, and D. Kilin, Dynamics of charge transfer and multiple exciton generation in the doped silicon quantum dot-carbon nanotube system: Density functional theory-based computation, J. Phys. Chem. Lett. 9, 5759 (2018).

[19] S. R. White, J. W. Wilkins, and K. G. Wilson, RenormalizationGroup Approach for Electronic Structure, Phys. Rev. Lett. 56, 412 (1986).

[20] J. Negele and H. Orland, Quantum Many-Particle Systems, 1st ed. (Addison-Wesley, Reading, MA, 1988).

[21] C. Gattringer and C. Lang, Quantum Chromodynamics on the Lattice: An Introductory Presentation, Lecture Notes in Physics (Springer, Berlin, 2009).

[22] D. Lee, Lattice methods and the nuclear few- and many-body problem, in An Advanced Course in Computational Nuclear Physics: Bridging the Scales from Quarks to Neutron Stars, edited by M. Hjorth-Jensen, M. P. Lombardo, and U. van Kolck (Springer International Publishing, Cham, 2017).

[23] D. J. Scalapino, A common thread: The pairing interaction for unconventional superconductors, Rev. Mod. Phys. 84, 1383 (2012).

[24] P. de Forcrand, Simulating QCD at finite density (2010), arXiv:1005.0539 [hep-lat].

[25] K. E. Schmidt and M. H. Kalos, in Applications of the Monte Carlo Method in Statistical Physics, edited by K. Binder (Springer-Verlag, Heidelberg, 1984).

[26] E. Y. Loh, J. E. Gubernatis, R. T. Scalettar, S. R. White, D. J. Scalapino, and R. L. Sugar, Sign problem in the numerical simulation of many-electron systems, Phys. Rev. B 41, 9301 (1990).

[27] S. Zhang and H. Krakauer, Quantum Monte Carlo Method using Phase-Free Random Walks with Slater Determinants, Phys. Rev. Lett. 90, 136401 (2003).

[28] B. Austin, D. Zubarev, and Jr. W. Lester, Quantum monte carlo and related approaches, Chem. Rev. 112, 263 (2012).

[29] S. Zhang, in Handbook of Materials Modeling, edited by W. Andreoni, S. Yip (Springer, Cham, 2018). 
[30] W. M. C. Foulkes, L. Mitas, R. J. Needs, and G. Rajagopal, Quantum Monte Carlo simulations of solids, Rev. Mod. Phys. 73, 33 (2001).

[31] S. Zhang, Finite-Temperature Monte Carlo Calculations for Systems with Fermions, Phys. Rev. Lett. 83, 2777 (1999).

[32] S. M. A. Rombouts, K. Heyde, and N. Jachowicz, Quantum Monte Carlo Method for Fermions, Free of Discretization Errors, Phys. Rev. Lett. 82, 4155 (1999).

[33] J. E. Drut and T. A. Lähde, Lattice field theory simulations of graphene, Phys. Rev. B 79, 165425 (2009).

[34] D. Smith and L. von Smekal, Monte-Carlo simulation of the tight-binding model of graphene with partially screened Coulomb interactions, Phys. Rev. B 89, 195429 (2014).

[35] M. V. Ulybyshev, P. V. Buividovich, M. I. Katsnelson, and M. I. Polikarpov, Monte-Carlo Study of the Semimetal-Insulator Phase Transition in Monolayer Graphene with Realistic InterElectron Interaction Potential, Phys. Rev. Lett. 111, 056801 (2013).

[36] Y. Otsuka, S. Yunoki, and S. Sorella, Universal Quantum Criticality in the Metal-Insulator Transition of Two-Dimensional Interacting Dirac Electrons, Phys. Rev. X 6, 011029 (2016).

[37] S. Beyl, F. Goth, and F. F. Assaad, Revisiting the Hybrid Quantum Monte Carlo Method for Hubbard and Electron-Phonon Models, Phys. Rev. B 97, 085144 (2018).

[38] W. Armour, S. Hands, and C. Strouthos, Monte Carlo simulation of monolayer graphene at nonzero temperature, Phys. Rev. B 84, 075123 (2011).

[39] T. Luu and T. A. Lähde, Quantum Monte Carlo calculations for carbon nanotubes, Phys. Rev. B 93, 155106 (2016).

[40] J. Ostmeyer, E. Berkowitz, S. Krieg, T. Lahde, T. A. Luu, and C. Urbach, Semimetal-Mmott insulator quantum phase transition of the Hubbard model on the honeycomb lattice, Phys. Rev. B 102, 245105 (2020).

[41] J. Phillips, Energy-Band Interpolation Scheme Based on a Pseudopotential, Phys. Rev. 112, 685 (1958).

[42] M. T. Yin and M. L. Cohen, Theory of $a b$ initio pseudopotential calculations, Phys. Rev. B 25, 7403 (1982).

[43] J. P. Perdew, K. Burke, and M. Ernzerhof, Generalized Gradient Approximation Made Simple, Phys. Rev. Lett. 77, 3865 (1996).

[44] S. Kümmel and L. Kronik, Orbital-dependent density functionals: Theory and applications, Rev. Mod. Phys. 80, 3 (2008).

[45] A. A. Abrikosov, L.P. Gorkov, and I. E. Dzyaloshinski, Methods of Quantum Field Theory in Statistical Physics (Prentice-Hall, Englewood Cliffs, NJ, 1963).

[46] A. L. Fetter and J.D. Walecka, Quantum Theory of ManyParticle Systems (McGraw-Hill, New York, 1971).

[47] L. Brown, Quantum Field Theory (Cambridge University Press, Cambridge, 1992).
[48] M. Govoni and G. Galli, Large scale gw calculations, J. Chem. Theory Comput. 11, 2680 (2015).

[49] R. Shankar, Principles of Quantum Mechanics (Plenum, New York, 1980).

[50] One recognizes that the approximate action [7] is not gauge invariant. However, the Coulomb gauge is already assumed in the KS equation. Full understanding of gauge invariance in this approach is left to future work.

[51] K. G. Wilson, Confinement of quarks, Phys. Rev. D 10, 2445 (1974).

[52] L.D. Landau and E.M. Lifshitz, Quantum Mechanics: NonRelativistic Theory, Course of Theoretical Physics (Elsevier Science, Amsterdam, 1981).

[53] Z. Fodor and S. D. Katz, A New method to study lattice QCD at finite temperature and chemical potential, Phys. Lett. B 534, 87 (2002).

[54] G. Aarts, Can Stochastic Quantization Evade the Sign Problem? The Relativistic Bose Gas at Finite Chemical Potential, Phys. Rev. Lett. 102, 131601 (2009).

[55] H. R. Kunsch, The jackknife and the bootstrap for general stationary observations, Ann. Statist. 17, 1217 (1989).

[56] P. Giannozzi, S. Baroni, N. Bonini, M. Calandra, R. Car, C. Cavazzoni, D. Ceresoli, G. Chiarotti, M. Cococcioni, I. Dabo, A. Dal Corso, S. de Gironcoli, S. Fabris, G. Fratesi, R. Gebauer, U. Gerstmann, C. Gougoussis, A. Kokalj, M. Lazzeri, L. Martin-Samos et al., Quantum espresso: a modular and opensource software project for quantum simulations of materials, J. Phys.: Cond. Matt. 21, 395502 (2009).

[57] D. R. Hamann, M. Schlüter, and C. Chiang, Norm-Conserving Pseudopotentials, Phys. Rev. Lett. 43, 1494 (1979).

[58] E. Anderson, Z. Bai, C. Bischof, S. Blackford, J. Demmel, J. Dongarra, J. Du Croz, A. Greenbaum, S. Hammarling, A. McKenney, and D. Sorensen, LAPACK Users' Guide, 3rd ed. (SIAM Publications, Philadelphia, 1999).

[59] R. B. Lehoucq, D. C. Sorensen, and C. Yang, ARPACK Users' Guide: Solution of Large-Scale Eigenvalue Problems with Implicitly Restarted Arnoldi Methods (SIAM Publications, Philadelphia, 1998).

[60] K. Wu, A. Canning, H. D. Simon, and L. w. Wang, Thickrestart Lanczos method for electronic structure calculations, J. Comput. Phys 154, 156 (1999).

[61] Z. W. Salsburg, J. D. Jacobson, W. Fickett, and W. W. Wood, Application of the monte carlo method to the lattice-gas model. I. Two-dimensional triangular lattice, J. Chem. Phys. 30, 65 (1959).

[62] A. M. Ferrenberg and R. H. Swendsen, New Monte Carlo Technique for Studying Phase Transitions, Phys. Rev. Lett. 61, 2635 (1988).

[63] S. Duane, A. D. Kennedy, B. J. Pendleton, and D. Roweth, Hybrid Monte Carlo, Phys. Lett. B 195, 216 (1987). 